J. Phys. IV France 138 (2006) 231-236

(C) EDP Sciences, Les Ulis

DOI: $10.1051 / \mathrm{jp} 4: 2006138026$

\title{
Métrologie d'indice par interférométrie EUV
}

\author{
S. de Rossi ${ }^{1}$, D. Joyeux ${ }^{1}$, F. Delmotte ${ }^{1}$ et B. Ea-Kim ${ }^{1}$ \\ ${ }^{1}$ Laboratoire Charles Fabry de l'Institut d'Optique, Campus de Polytechnique, \\ RD 128, 91127 Palaiseau, France
}

\begin{abstract}
Résumé. Nous décrivons la détermination directe des indices de matériaux en fonction de la longueur d'onde par interférométrie dans la gamme spectrale EUV (10-50 nm). L'interféromètre de type division de front d'onde est un bimiroir de Fresnel. Le principe consiste à mesurer le décalage des franges entre le côté de l'interférogramme qui a vu l'échantillon et le côté référence sans échantillon. Une analyse de Fourier permet d'extraire l'épaisseur optique du matériau. Nous présentons les premiers résultats de la mesure d'indice du molybdène avec l'instrument réalisé.
\end{abstract}

\section{INTRODUCTION}

Connaître l'indice d'un matériau, c'est connaître l'interaction lumière-matière d'un point de vue macroscopique. Il s'avère alors indispensable dans la science des matériaux de déterminer de manière précise cette propriété optique fondamentale. Alors que le domaine de l'optique EUV connaît une évolution rapide depuis une dizaine d'années avec le développement de nouvelles sources EUV, la connaissance précise des indices reste tout aussi indispensable pour la réalisation de composants performants associés aux sources. En effet, les techniques optiques habituellement utilisées dans d'autres domaines spectraux (de l'UV à l'IR) diffusent vers l'EUV pour donner naissance à de nouveaux composants (miroir haute réflectivité, miroir large bande, miroir déphasant, lame séparatrice, polariseur), essentiellement basés sur des revêtements multicouches, dont les techniques de dépôt sont maintenant bien contrôlées. Dans le domaine EUV, il est usuel d'écrire l'indice sous la forme :

$$
\mathrm{n}(\lambda)=1-\delta(\lambda)+\mathrm{i} \beta(\lambda)
$$

où la partie réelle $1-\delta$ (composante réfractive) et la partie imaginaire $\beta$ (composante absorbante) sont très fortement dépendantes de la longueur d'onde. Les méthodes habituellement utilisées pour la détermination de l'indice sont basées soit sur des mesures d'absorption par application des relations de Kramers-Kronig [1], soit sur des mesures de réflectivité de multicouches ou de monocouches [2]. Dans le premier cas, bien que les relations utilisées soient rigoureuses, leurs mises en oeuvre exactes exigeraient des mesures d'absorption très précises et nombreuses, jamais vraiment réalisées de façon satisfaisante. De ce fait, le résultat des calculs est en pratique sujet à caution, surtout dans les régions les plus intéressantes du spectre, au voisinage des seuils d'absorption. Dans le deuxième cas, les déterminations sont encore fortement perturbées par l'absorption du matériau, ainsi que par la rugosité des couches. Dans les deux cas, l'extraction de l'indice n'est pas directe, et nécessite un traitement numérique plus ou moins lourd, voire l'introduction de modèles mathématiques (seuils d'absorption ou rugosité). De plus, la plupart des laboratoires s'intéressant à cette thématique ne disposent pas forcément des outils nécessaires à la réalisation de composants optiques EUV. Par conséquent, les matériaux mesurés ne sont pas nécessairement élaborés avec les techniques de dépôts ni dans des conditions usuelles mises en oeuvre dans la réalisation des optiques (notamment de vide résiduel).

Le caractère indirect de ces déterminations expérimentales, l'utilisation de simulations diverses et la variété des conditions expérimentales utilisées impliquent des dispersions des mesures entre les différentes valeurs trouvées dans la littérature. En exemple, la figure 1 montre la partie réelle et la partie imaginaire de l'indice entre 10 et $50 \mathrm{~nm}$ pour le molybdène. 


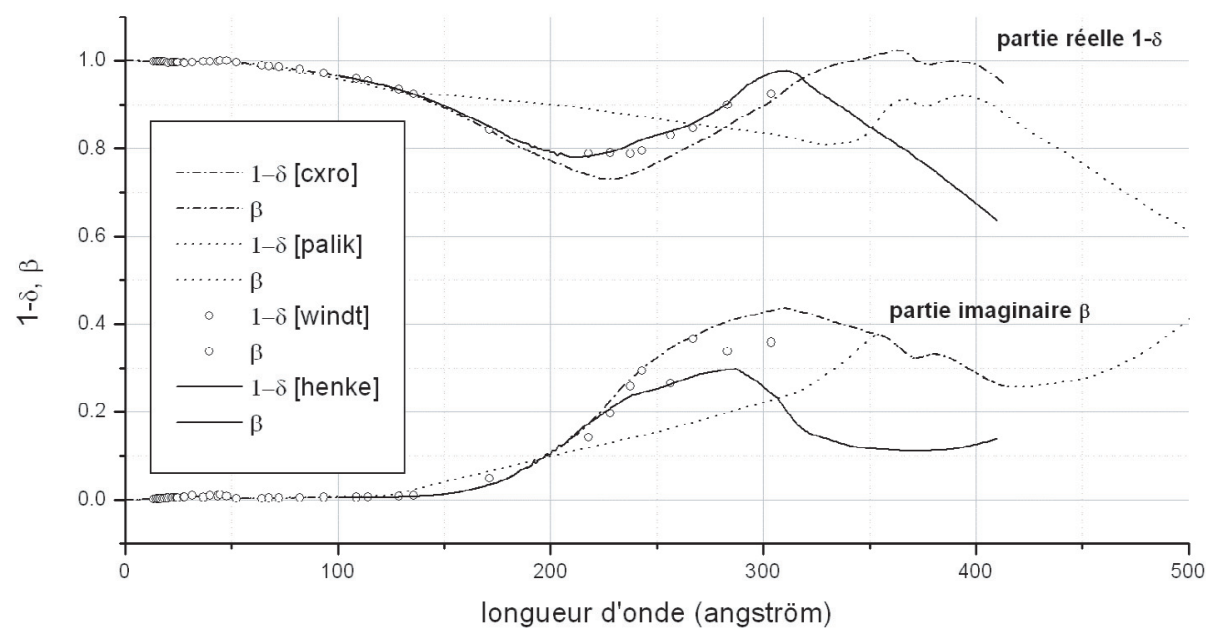

Figure 1. Evolution de la partie réelle et imaginaire de l'indice complexe du molybdène déterminé par différentes méthodes et auteurs. $[\mathrm{cxro}]=[3] /[$ palik $]=[4] /[$ windt $]=[5] /[$ henke $]=[6]$.

Pour répondre à ce besoin croissant de la connaissance précise des indices optiques dans le domaine EUV, l'interférométrie, outil métrologique par excellence pour la détermination de la phase (épaisseur optique), donne un accès direct à la partie réelle de l'indice et à ses variations en fonction de n'importe quel paramètre physique (longueur d'onde en particulier). Une seule mesure sur un échantillon mince en transmission suffit à déterminer un indice à une longueur d'onde, par une simple règle de 3 . En effet dans un interféromètre à deux ondes, l'introduction d'un échantillon mince transmissif d'épaisseur e et d'indice $1-\delta$ dans un des bras (l'autre étant idéalement le vide) induit un décalage des franges d'une quantité $\Delta=\delta \mathrm{e} / \lambda$. De plus, en transmission et en incidence normale, on peut montrer que la perturbation par d'éventuelles réflexions parasites aux interfaces est négligeable, du fait de la relative faiblesse de $\delta$.

\section{L'INTERFÉROMÈTRE}

Faire de l'interférométrie aux courtes longueurs d'onde n'est pas aussi simple que dans le visible. Les fortes absorptions des matériaux affaiblissent l'utilisation de lames séparatrices (interféromètre à division d'amplitude), bien que des travaux aient été menés dans ce sens à l'Institut d'Optique [7] et dans d'autres équipes [8]. De plus les lames réalisées, toujours basées sur des multicouches, sont fortement chromatiques, ce qui est gênant pour des mesures en fonction de la longueur d'onde. La réalisation de composants diffractifs contourne l'utilisation des multicouches mais ils restent tout aussi chromatiques et limitent donc la mesure autour d'une longueur d'onde d'intérêt [9].

Pour contourner cette difficulté et permettre un plus grand choix d'applications, Denis Joyeux et al ont proposé au début des années 90 l'utilisation d'interféromètres à division de front d'onde de type bimiroir de Fresnel, qui par principe n'utilisent pas de lames séparatrices. En travaillant en incidence plus ou moins rasante sur des miroirs plans, l'outil peut travailler sur une grande plage de longueur d'onde. De plus la nécessité d'une source de luminance élevée et accordable en longueur d'onde a imposé les sources synchrotron, rendant possible une véritable mesure de la dispersion des indices. En 1992, la source du LURE à Orsay a permis de valider le principe de la mesure autour du seuil d'absorption $K \alpha$ du carbone sur des échantillons en transmission [10].

L'interféromètre utilisé pour la détermination des indices est un bimiroir de Fresnel (figure 2). Il est constitué de deux blocs de silice superpolis de rugosité de l'ordre de $0,1 \mathrm{~nm}$ et de planéité meilleure que $\lambda / 100$ dans la zone utile. Il est éclairé sous $6^{\circ}$ d'incidence rasante. Sa réflectivité reste alors élevée 


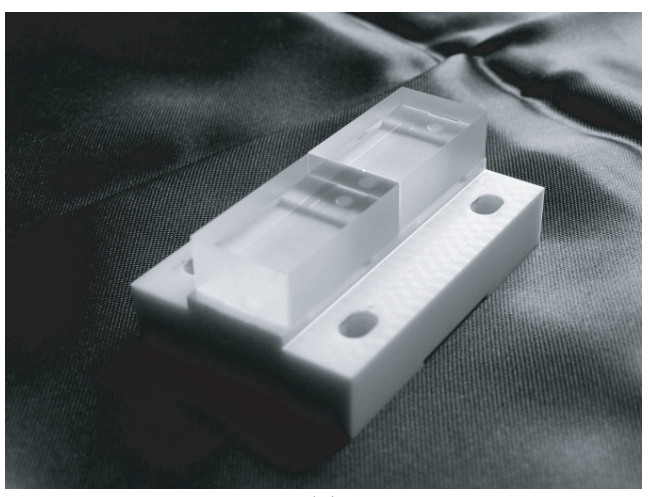

(a)

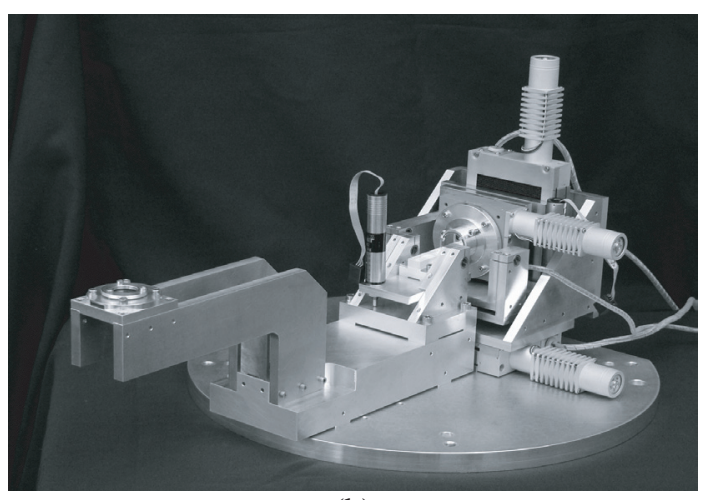

(b)

Figure 2. Bimiroir de Fresnel collé sur une embase en céramique (a) et mécanique de déplacement des différents éléments de l'interféromètre (b).

sur la plage 10-50 $\mathrm{nm}$. L'angle entre les deux miroirs est de 0,85 mrad. Il impose un interfrange à $30 \mathrm{~nm}$ de $18 \mu \mathrm{m}$ et un champ de franges observable à $40 \mathrm{~cm}$ de $680 \mu \mathrm{m}$. Pour faciliter la détection des franges et augmenter d'un facteur 10 l'interfrange et le champ, le plan de détection est inclinée de $6^{\circ}$ rasant par rapport à la directrice du faisceau $\left(90^{\circ}\right.$ dans le cas d'une détection normale). Dans ces conditions et pour la plage de longueur d'onde voulue la figure d'interférence contient quelques dizaines de franges avec un interfrange apparent supérieur à $50 \mu \mathrm{m}$, ce qui satisfait pleinement le critère d'échantillonnage. Le détecteur est un cristal de YAG (placé sous vide) qui fluoresce dans le visible. Une caméra CCD visible couplée à un système d'imagerie (placé dans l'air) permet de visualiser et enregistrer les interférogrammes.

L'interféromètre est réglé par construction pour que la frange blanche soit centrée dans le champ lors d'un éclairage large bande, permettant de s'affranchir au maximum de l'effet de cohérence temporelle de la source (réglage à la différence de marche nulle). Un interféromètre à division de front d'onde nécessite de plus un certain degré de cohérence spatiale de la source, qui dépend du champ de frange désiré et du flux accessible. En d'autre terme, nous requérons une luminance spectrale suffisante (éclairement cohérent supérieur à $10^{11}$ photons. $\mathrm{s}^{-1} \cdot \mathrm{cm}^{-2}$ pour une bande passante inférieure au \%) pour éviter des temps de pause trop long sur un champ trop petit. Le champ est alors simplement fixé par la distance entre la source et l'interféromètre. Les sources synchrotrons de $3^{\text {ème }}$ génération offrent des luminances spectrales suffisantes et permettent ainsi une détection faible bruit pour des temps de pause raisonnables.

\section{LA TECHNIQUE}

Afin de déterminer le déphasage introduit par un échantillon mince transmissif, il est nécessaire de comparer le système de franges associé à l'échantillon avec un système de franges référence. Pour permettre une mesure directe, la référence et l'échantillon seront placés en même temps dans l'interféromètre.

Le principe de la mesure consiste à venir éclairer avec un faisceau EUV un échantillon dont un quart du champ est le matériau dont on désire mesurer l'indice (figure 3). Les trois quart restant sont soit le vide, mais cela implique quelques difficultés pratiques, soit une membrane de support que l'on fera la plus fine possible. Si on s'arrange pour venir éclairer un des miroirs du bimiroir par la moitié supérieure du champ (quart du matériau et quart membrane) et la moitié inférieure du champ sur l'autre miroir (deux fois le quart de la membrane), le croisement des faisceaux réfléchis par le bimiroir sera le lieu de deux systèmes de franges : l'un étant lié à l'interférence entre l'onde transmise par le matériau+membrane et l'onde transmise par la membrane uniquement, et l'autre par la membrane avec 


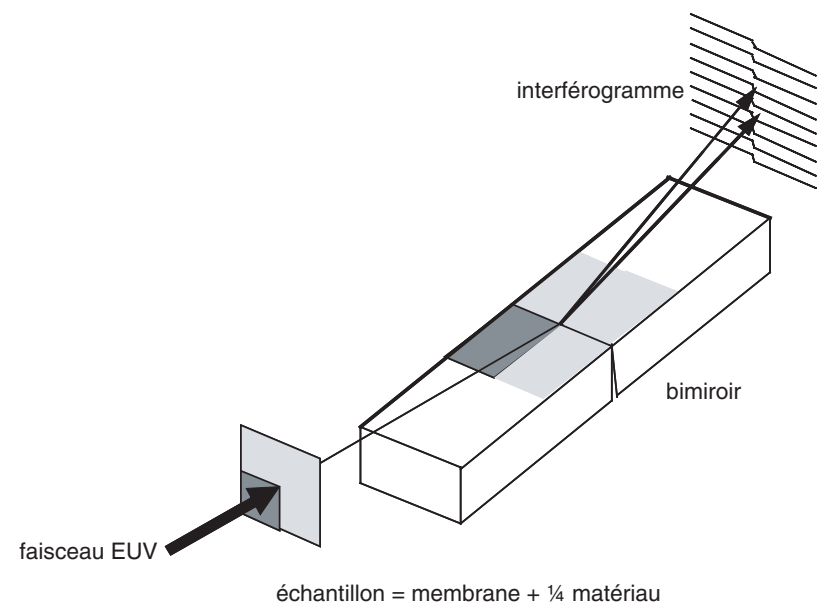

Figure 3. Principe de la mesure d'indice par interférométrie EUV avec un bimiroir de Fresnel.

elle-même. Les deux systèmes de franges sont donc parallèles et décalés d'une quantité proportionnelle à l'épaisseur optique du matériau analysé (produit de son épaisseur et de son indice de réfraction à la longueur d'onde de travail). La mesure de l'épaisseur est obtenue par réflectométrie en X rasants fonctionnant à $0,154 \mathrm{~nm}$ avec des précisions sur l'épaisseur de $0,2 \mathrm{~nm}$.

\section{LES ÉCHANTILLONS}

L'échantillon est une membrane ultrafine de nitrure de silicium recouverte de matériau à analyser sur un quart de la membrane (figure $4 b$ ). Les résultats préliminaires ont été réalisés avec du molybdène. Le nitrure de silicium est obtenu par dépôt sur une galette de silicium par PECVD (Plasma Enhanced Chemical Vapour Deposition) avec contrôle de la contrainte. Une gravure humide face arrière du silicium permet de libérer les membranes. Par masquage et photolithographie UV, les trois quarts de la membrane sont recouverts de résine. Le molybdène est ensuite déposé par pulvérisation cathodique magnétron. Une densité moyenne de $10,1 \pm 0,8 \mathrm{~g} \cdot \mathrm{cm}^{-3}$ pour le molybdène a été mesurée par RBS (analyse par rétrodiffusion coulombienne). Cette densité est voisine de la densité théorique de $10,2 \mathrm{~g} \mathrm{~cm}^{-3}$ pour le molybdène cristallin massif. La dissolution de la résine permet de révéler le quart de molybdène sur la membrane (technique dite de « lift off »). Le matériau est déposé dans les mêmes conditions que l'élaboration de multicouches (miroirs Mo/Si par exemple). Un amincissement de la membrane par gravure ionique réactive (RIE) permet de passer d'une épaisseur de $500 \mathrm{~nm}$ à $50 \mathrm{~nm}$. Dans ces conditions l'échantillon transmet $10 \%$ à $\lambda=20 \mathrm{~nm}$ et moins de $2 \%$ à $\lambda=30 \mathrm{~nm}$. Une fine couche de passivation (silicium ou autre) de quelques nanomètres pourra être déposée pour éviter l'oxydation du matériau.

\section{L'ANALYSE DES INTERFÉROGRAMMES}

L'analyse des interférogrammes est basée sur les propriétés classiques de la transformée de Fourier. Il est bien connu que la transformée de Fourier complexe uni-dimensionnelle d'un signal sinusoïdal donne un pic à la fréquence du signal. La phase de ce pic mesure la position de la sinusoïde par rapport à une origine dans l'espace réel. On peut appliquer cette détermination à l'interférogramme, ligne par ligne à peu près perpendiculaire aux franges. La variation de phase est alors linéaire et continue. En présence d'un échantillon, un saut de phase est présent à l'interface référence / matériau (figure 4). La mesure du saut de phase par ajustement linéaire (au sens des moindres carrés) des deux droites de phase 


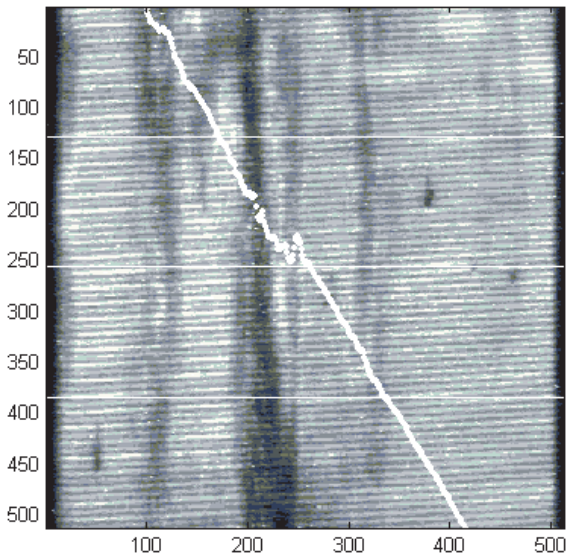

(a)

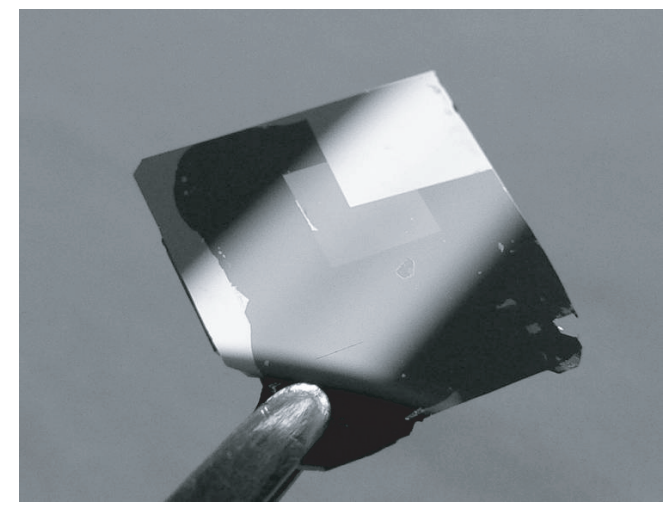

(b)

Figure 4. (a) Interférogramme à $22 \mathrm{~nm}$ avec sa phase en ligne. Le décrochement de la phase est bien visible et vaut ici $1 / 16$ de frange. Les zones sombres sont des résidus de résine très absorbants. (b) Photo de la membrane de nitrure de silicium de $50 \mathrm{~nm}$ avec $20 \mathrm{~nm}$ de molybdène.

(avec contrainte de parallélisme) permet donc de déterminer l'épaisseur optique du matériau. Cette technique d'analyse permet d'obtenir des précisions sur le décalage de l'ordre de $\lambda / 10000$ dans le cas idéal d'interférogramme calculé et sans bruit. La précision sur le décalage dépend donc essentiellement du bruit de la mesure, qui se décline sous plusieurs aspects : l'uniformité de l'échantillon, la planéité des miroirs et enfin le bruit de la caméra. La prise en compte de toutes ses sources de bruit permet au final d'obtenir une précision meilleur que 1/100 de franges.

\section{LES RÉSULTATS PRÉLIMINAIRES}

Nous présentons les premiers résultats obtenus avec cet interféromètre pour le molybdène dans la gamme spectrale 10-30 nm. En raison des très fortes absorptions de ce matériau les mesures deviennent très difficiles après $30 \mathrm{~nm}$ avec les échantillons réalisés. Nous avons effectués deux séries de mesures sur la ligne XIL du synchrotron Suisse à l'Institut Paul Scherrer. L'éclairement cohérent suffisant (à $13,6 \mathrm{~nm}, \mathrm{E}=2 \cdot 10^{13}$ photons. $\mathrm{s}^{-1} \cdot \mathrm{cm}^{-2}$ pour $0,09 \%$ de bande passante, un courant de $0,3 \mathrm{~A}$ et un faisceau de dimension $4 \mathrm{~mm}$ x $4 \mathrm{~mm}$ ) et la possibilité de travailler à une dizaine de mètres de la fente de sortie du monochromateur permettent d'obtenir un champ convenable et des temps de pause relativement court (quelques minutes au maximum).

Sur la figure 5, la série de mesure 1 représente l'évolution de l'indice pour du molybdène non protégé de l'oxydation. La série de mesure 2 représente un échantillon protégé par une fine couche de silicium (quelques nanomètres) rapidement oxydé. Les mesures des deux séries concordent bien avec les mesures effectuées par d'autres techniques. En revanche il apparaît un décalage net aux courtes longueurs d'onde entre l'échantillon protégé (indice plus élevé) et non protégé. La formation d'un composé de siliciure de molybdène à l'interface molybdène-silicium pourrait expliquer ce décalage. L'indice mesuré est donc un indice effectif qui dépend du taux d'interdiffusion à l'interface. Pour remédier à ce problème, il est nécessaire, soit de mesurer l'échantillon in situ, soit de protéger le molybdène par un autre composé moins réactif. Le carbure de bore pourrait être une piste à étudier. En revanche l'échantillon non protégé ne présente pas une différence notable avec les autres techniques. Soit l'oxydation est faible, soit les échantillons utilisés par les autres auteurs étaient oxydés dans les mêmes proportions. Cette hypothèse est la plus probable, car le phénomène d'oxydation est considéré comme un phénomène de surface rapide. 


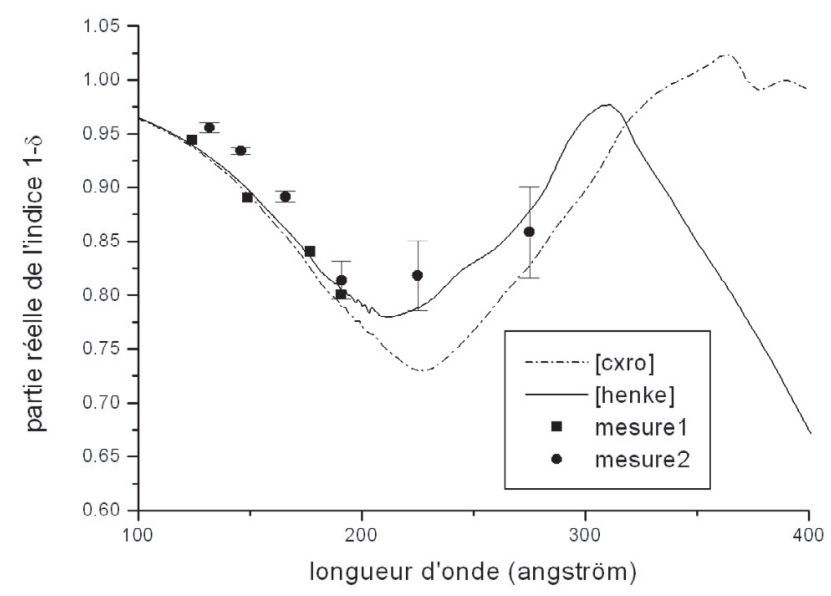

Figure 5. Mesure de la partie réelle de l'indice en fonction de la longueur d'onde par interférométrie EUV. Comparaison avec les mesures tabulées par le CXRO [3] et obtenues par Henke [6].

\section{Remerciements}

Les auteurs remercient Daniel Phalippou pour son savoir faire unique dans la réalisation mécanique de l'interféromètre et Gilles Colas pour son doigté inégalé dans le polissage des miroirs.

\section{Références}

[1] R. de L. Kronig et H.A. Kramers, "La diffusion de la lumière par les atomes", Atti Congr. Inter. Fisici Como 2 (1927) 545-557.

[2] R. Soufli and E. Gullikson, "Reflectance measurements on clean surface for the determination of optical constants of silicon in the extreme ultraviolet-soft-x-ray region", Appl. Opt. 36 (1997) 5499-5507.

[3] http://www.cxro.lbl.gov/optical_constants/

[4] E. D. Palik, Ed., Handbook of Optical Constants of Solids (Academic, Orlando, FL) (1985).

[5] D. L. Windt, "XUV optical constants of sigle-crytal GaAs and sputtered C, Si, Cr3C2, Mo and W", Appl. Opt. 30 (1991) 15-25.

[6] B. L. Henke, E. M. Gullikson and J. C. Davis, "X-ray interactions: photabsorption, scattering, transmission and reflection at E=50-30000 eV, Z=1-92", At. Data Nucl. Data Tables 54 (1993) 181-343.

[7] F. Delmotte et al., "X-ray-ultraviolet beam splitters for the Michelson interferometer", Appl. Opt. 41(28) (2002) 5905-5912.

[8] L. B. Da Silva et al, "Extreme-ultraviolet interferometry at 15,5 nm using multilayer optics", Appl. Opt. 34(28) (1995) 6389-6392.

[9] K. Rosfjord, C. Chang, R. Miyakawa, H. Barth and D. Attwood, "Direct index of refraction measurements at extreme-ultraviolet and soft-x-ray wavelength", Appl. Opt. 45(8) (2006) 17301736.

[10] D. Joyeux, F. Polack, D. Phalippou, "An interferometric determination of the refractive part of optical constants for carbon and silver across soft x-ray absorption edges", Rev. Sci. Instrum. 70(7) (1999) 2921-2926. 